\title{
Signos radiológicos: Esófago felino
}

\author{
Drs. Gonzalo Pérez $\mathrm{O}^{(1)}$, Mario Zerega $\boldsymbol{R}^{(1)}$, Claudio Cortés $A^{(2)}$.
}

1. Residente de Radiología, Centro de Imagenología, Hospital Clínico Universidad de Chile. Santiago, Chile.

2. Radiólogo, Profesor Asociado Facultad de Medicina, Universidad de Chile. Centro de Imagenología, Hospital Clínico Universidad de Chile. Santiago, Chile.

\section{Radiological signs: Feline esophagus}

Abstract: Feline esophagus is the benign transient fine circumferential transverse folds that arise during double-contrast esophagography. Accurate discrimination of this entity is relevant due to its association with gastroesophageal reflux and hiatal hernia. Among the differential diagnoses, fixed folds secondary to reflux esophagitis and eosinophilic esophagitis have to be considered.

Key words: Esophagus, Fluoroscopy, Gastroesophageal reflux, Radiology.

Resumen: El signo del esófago felino corresponde a múltiple pliegues finos, dispuestos transversalmente, muy cercanos y generalmente transitorios, detectados en el estudio con doble contraste del esófago. Su reconocimiento es importante por su asociación con reflujo gastroesofágico y hernia hiatal. Dentro de los diagnósticos diferenciales a considerar se encuentran los pliegues fijos secundarios a esofagitis por reflujo y la esofagitis eosinofílica.

Palabras claves: Esófago, Fluoroscopía, Reflujo gastroesofágico, Radiología.

Pérez G y cols. Signos radiológicos: Esófago felino. Rev Radiol 2012; 18(1): 36-38.

Correspondencia: Dr. Gonzalo Pérez O. / gnperez@gmail.com

Trabajo recibido el 05 de marzo de 2012, aceptado para publicación el 19 de marzo de 2012.

\section{Aspecto imaginológico}

El signo del esófago felino corresponde a la visualización, en una esofagografía de doble contraste, de múltiples pliegues finos, habitualmente de 1 a $2 \mathrm{~mm}$ de espesor, que se extienden de forma transversal por el esófago. Se disponen muy próximos entre sí y habitualmente corresponden a un fenómeno transitorio, detectado ya sea durante la deglución del contraste o en algún episodio de reflujo ${ }^{(1)}$. Aunque se pueden detectar en cualquier segmento, su localización más frecuente corresponde al tercio inferior del esófago (Figuras 1 y 2 ).

Figura 1. Esofagografía de doble contraste. Se observan en tercio inferior los pliegues clásicos del esófago felino durante la deglución de contraste, en una paciente con reflujo gastroesofágico severo.

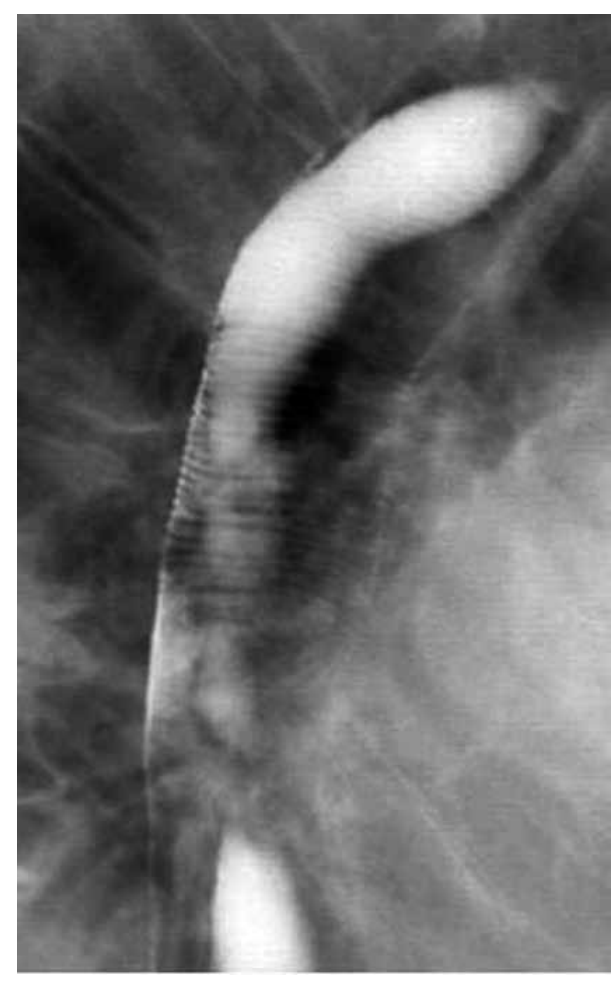




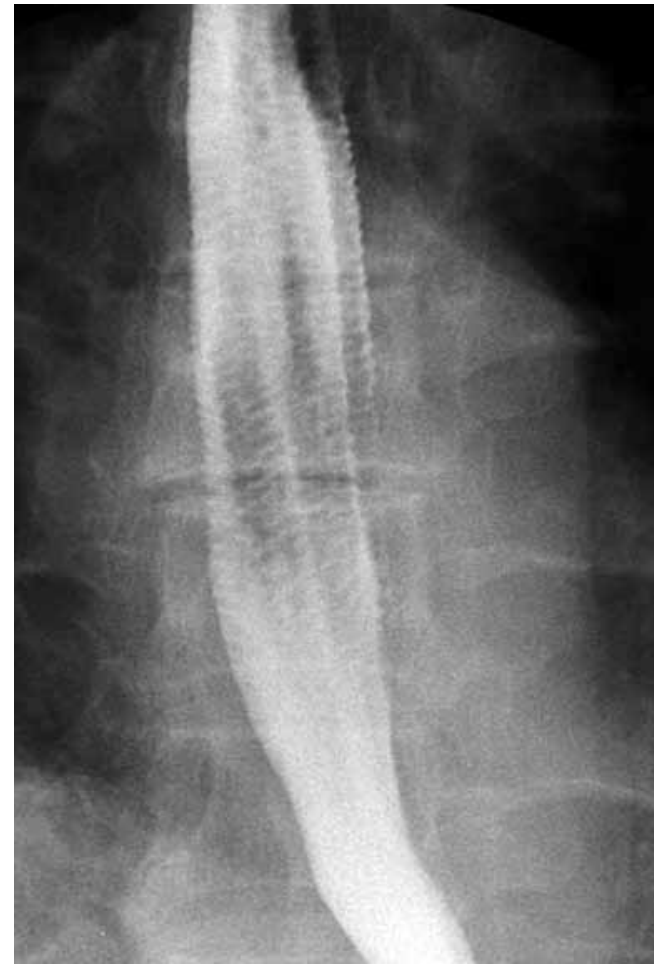

Figura 2. Esofagografía de doble contraste. Pliegues transversales finos constituyendo el signo del esófago felino durante un episodio de reflujo.

\section{Reseña histórica}

Los pliegues esofágicos transversales fueron descritos por primera vez como parte de la anatomía normal de gatos por Bremmer et al(2) y Goldberg et $\mathrm{al}^{\left({ }^{(3)}\right.}$ de forma casi simultánea, en 1970. En el estudio de Goldberg se demostraron estos pliegues mediante esofagografías con contraste, utilizando polvo de Tantalio. Se describieron como pliegues fijos, que cubrían toda la circunferencia del esófago. Ya desde entonces hay evidencia de pliegues transversales similares, ocasionalmente visualizados en pacientes sometidos a estudios con doble contraste. Dado la similitud con el patrón presentado en los gatos (Figura 3 ), se ha denominado signo del esófago felino.

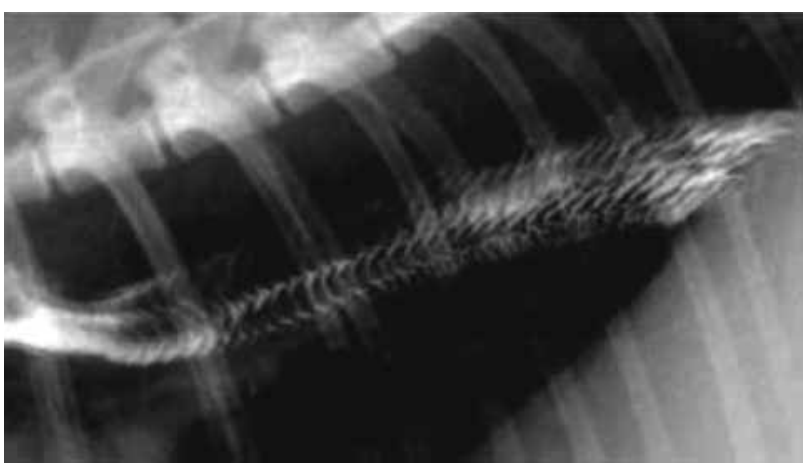

Figura 3. Estudio con contraste de tracto digestivo alto en un gato. Pliegues transversales normales a lo largo del segmento esofágico visualizado.

\section{Significado}

El esófago está formado por una capa mucosa (que consta de epitelio, lámina propia y muscular de la mucosa), por la capa submucosa y por la muscular externa. Son los componentes de la mucosa los determinantes en la fisiopatología del signo del esófago felino. Específicamente, el mecanismo por el cual se produce ha sido atribuido a la contracción de las fibras longitudinales de la muscular de la mucosa, que permite que el epitelio y tejido conectivo que recubre se pliegue de forma transversa en el área comprometi$\mathrm{da}^{(4)}$. Aunque no está del todo demostrado, la mayoría de los autores coincide en que es este fenómeno el responsable de las características descritas.

Su importancia radica en la asociación que presenta con el reflujo gastroesofágico. Williams et al. ${ }^{(4)}$ en 1983 demostraron la importante relación existente $y$, ya entonces, sugirieron el estudio de reflujo y sus complicaciones, incluso en pacientes asintomáticos en que se observara este signo. En un estudio más reciente, Samadi et al(5) confirmó los hallazgos demostrando reflujo gastroesofágico en todos los pacientes estudiados con esófago felino. Más aún, se demostró que también existía correlación entre el grado de reflujo y su presencia, siendo más frecuente con la patología severa. También se ha establecido asociación con la hernia hiatal(5,6). Aunque de menor magnitud que con el reflujo, se demostró que los pacientes con esófago felino presentaban más frecuentemente esta condición.

Dado que es conocida la baja sensibilidad de los estudios contrastados de tracto digestivo alto para el diagnóstico de reflujo gastroesofágico $(70 \%$ incluso con maniobras de provocación) ${ }^{(7)}$, es valioso reconocer e interpretar de forma correcta el signo de esófago felino.

\section{Diagnóstico diferencial}

El principal diagnóstico diferencial a plantear corresponde a los pliegues fijos producidos en el esófago distal por esofagitis secundaria a reflujo. Estos pliegues generalmente son más gruesos (de 2 a $5 \mathrm{~mm}$ ), no rodean por completo el esófago, están más separados y son persistentes durante la fluoroscopía $^{(8)}$. Otro diagnóstico diferencial es la esofagitis eosinofílica idiopática que se suele manifestar como indentaciones en forma de anillo, casi siempre asociado a diversos grados de estenosis ${ }^{(9)}$.

Si bien estos diagnósticos se deben considerar, una revisión cuidadosa de sus características permite su diferenciación en el estudio esofágico de doble contraste con el signo de esófago felino.

\section{Discusión}

El reflujo gastroesofágico es una condición sumamente frecuente, que deteriora -en muchos casos- de forma importante la calidad de vida de los 
pacientes y que presenta complicaciones a corto y largo plazo. Entre estas últimas se incluyen algunas muy severas como el esófago de Barrett y el adenocarcinoma esofágico, por lo que su diagnóstico precoz es fundamental para un manejo adecuado. Si bien el gold estándar para su detección es la pHmetría de 24 horas, los estudios contrastados de tracto digestivo alto también pueden aportar en el diagnóstico y en el reconocimiento de las complicaciones. El valor del signo del esófago felino radica en la ayuda que presta para el diagnóstico. Está bien documentada su asociación con esta condición y presenta una apariencia distintiva en los exámenes de doble contraste, por lo que puede ser utilizado como un signo indirecto de reflujo. Más aún, en base a los resultados de los últimos estudios, incluso podría orientar sobre el grado de reflujo presente ${ }^{(5)}$. Para este propósito es fundamental realizar un examen óptimo y conocer las características del signo.

\section{Bibliografía}

1. GohelVK, Edell SL, Laufer I, Rhodes WH. Transverse folds in the human esophagus. Radiology 1978: 128: 303-308.
2. Bremner CG, Shorter RG, Ellis FH. Anatomy of feline esophagus with special reference to its muscular wall and phrenoesophageal membrane. J Surg Res 1970; 10: 327-331.

3. Goldberg HI, Dodds WJ, Jenis JH. Experimental esophagitis: roentgenographic findings after insufflation oftantalum powder. AJR 1970; 110: 288-294.

4. Williams SM, Harned RK, Kaplan P., Consigny PM. Work in progress: transverse striations of the esophagusassociation with gastroesophageal reflux. Radiology 1983; 146: 25-27.

5. Samad F, Levine M, Rubesin S, Katzka D, Laufer I. Feline Esophagus andGastroesophageal Reflux, AJR 2010; 194: 972-976.

6. Furth EE, Rubesin SE, Rose D. Feline esophagus. AJR 1995; 164: 900.

7. Thompson JK, Koehler RE, Richter JE. Detection of gastroesophageal reflux: value of barium studies compared with 24-hr pH monitoring. AJR 1994; 162: 621-626.

8. Levine MS, Goldstein HM. Fixed transverse folds in the esophagus: a sign of reflux esophagitis. AJR 1984; 143: 275-278.

9. Zimmerman SL, Levine MS, Rubesin SE et al. Idiopathic eosinophilic esophagitis in adults: the ringed esophagus. Radiology 2005; 236: 159-165. 